\title{
Factors associated with citation rate of randomised controlled trials in physiotherapy
}

\author{
Matteo Paci ${ }^{*}$, Niccolò Landi ${ }^{2}$, Gennaro Briganti ${ }^{3}$ and Bruna Lombardi ${ }^{1}$
}

\begin{abstract}
Background: Despite the use of citation rate as a measure of quality of research is strongly criticized and debated, it remain a widely used method to evaluate performances of researchers, articles and journals. The aim of this study was to test which factors are associated with citation rate of Randomised Controlled Trials (RCTs) published on the physiotherapy field.

Methods: All RCTs abstracted in the Physiotherapy Evidence Database (PEDro), indexed in Scopus database and published in 2008 were included. PEDro score, language of publication, indexing in PubMed database, type of access to articles, subdiscipline, the number of authors, the country where the study was performed, the type of institution where the study was conducted and the number of centres involved in the study (multicentric vs singlecentre). and the 2013 5-year impact factor of the publishing journals were considered as independent variables. Citation rate until December 2013 was extracted from Scopus database and used as dependent variable.

Results: Six hundred and nineteen RCTs, published in 283 journals, were included and analysed. The 5-year impact factor was the strongest variable associated with the citation rate and explained approximately $50 \%$ of the variance, and the number of authors explained an additional small part (about $1 \%$ ) of variability. The other variables were excluded from the model.

Conclusions: The study highlights that 5-year Impact Factor, not accessibility (language of publication, indexing in PubMed database or the type of access to articles) or reported quality (PEDro score), is a strong predictor of the number of citations for RCTs in the physiotherapy field. Our findings support the increasingly widespread idea that citation analysis does not reflect the scientific merit of the cited work, at least in terms of reported quality.

The results of this study need to be confirmed with a publication window larger than one year.
\end{abstract}

Keywords: Randomised controlled trials, Quality ratings, Physiotherapy, Bibliometrics, Periodicals

\section{Background}

The dissemination of research findings requires publication in peer-reviewed journals, and it is frequently measured through citation of the original work in subsequent publications. However, it is recognized that many factors outside the pure merit of the research or the authors influence such counts and many authors doubts that citation counts can really reflect the impact of scientific activity. The main reported sources of bias are self-citations, the

\footnotetext{
* Correspondence: matteo.paci@applicazione.it

'Unit of Functional Rehabilitation, Department of Continuing Care, Azienda USL 4, Prato, Italy

Full list of author information is available at the end of the article
}

increase in the total number of citations with time, or the correlation between the number of authors of an article and the number of citations it receives [1]. Moreover, citations are not necessarily representative of articles value, since papers can also be cited to be criticized.

A number of different indicators has been suggested to assess outcomes of biomedical research. In a recent review, Thonon et al. [2] found a total of 57 indicators aimed to assess research activity, collaboration, industrial production, dissemination and health service impact, in addition to scientific production and impact.

Anyway, citation analysis remain popular to evaluate performances of researchers, journals, universities, up to 
whole countries [3]. Citation count is also currently used as one of the criteria to select university professors in Italy [4] and for allocation of university research funds in many countries [5].

In addition, the number of citations received by an article is frequently viewed by researchers as the most useful measure of impact $[6,7]$. It can be supposed that the number of citations received by an article should be related to its methodological quality and relevance of the issue. However, it also may be influenced by other factors, like accessibility to both database and full text, or language of publication.

A number of authors examined the citation rate of original researches in different fields of medicine [8-12]. However, articles with different study designs were included and, in most of the cases, the study design was found to be a strong predictor of citation rate $[10,11]$. Randomised controlled trials (RCTs) are traditionally considered the gold standard for examining the efficacy of interventions. In the field of physiotherapy, RCTs represent a small part of the totality of research articles, but their rate is comparable to other disciplines [13-15]. The number of RCTs published in physiotherapy journals [14] and the quality of RCTs in physiotherapy field [16-18] show an increase over years. To our knowledge, no studies have been performed to specifically analyse the citation rate of this relevant type of articles.

The aim of this study was to test which factors are associated with citation rate of RCTs published within the physiotherapy field.

\section{Methods}

All RCTs abstracted in the Physiotherapy Evidence Database (PEDro), indexed in Scopus database and published in 2008 were included. Article title, journal name, year of publication, ratings for each of the 11 items of the PEDro scale, and total PEDro score of all RCTs that had complete PEDro scale ratings were downloaded from PEDro database, a free database of RCTs, systematic reviews and clinical practice guidelines in physiotherapy. In addition to the PEDro score, the following variables were recorded for each article: (a) the language of publication (English vs other languages), (b) indexing in PubMed database, (c) the type of access to articles (open access, delayed open access or restricted access), (d) the subdiscipline; (e) the 2013 5-year Impact Factor of the journals where the articles were published; $(f)$ the number of authors; (g) the country where the study was performed; (h) the type of institution where the study was conducted (academic vs non- academic) and (i) the number of centres involved in the study (multicentric vs single-centre).

Citation rate until December 2013 was extracted from Scopus database.

\section{Type of access}

Access to the articles was defined as "open" when they were immediately available for free at the time of publication. Differently, they were defined as "delayed open" when they were available for free not immediately (e.g. after 6 months or 1 year). Finally, articles were considered as having "restricted access" when they were available only for subscribers.

\section{RCT Quality score (PEDro score)}

The PEDro database provides the assessment of the reported methodological quality of each RCT using the PEDro scale $[19,20]$, which rates 11 aspects of methodological quality of RCTs as being either absent or present. The first item (eligibility criteria) is not scored because it does not relate to internal validity or statistical reporting, therefore the total score ranges from 10 (RCT that satisfies all criteria) to 0 (RCT that does not satisfy any of the criteria). The PEDro Scale total score has been shown to be reliable for use in systematic reviews of physical therapy RCTs [21]. There is evidence for its construct validity [22], convergent and construct validity [23], discriminative validity, face validity, and content validity [24].

\section{Subdisciplines}

Subdiscipline classification was based on PEDro database classification. However, since a number of articles may be classified in different categories, clinical categories were preferred in classification process. For example, articles were included in Paediatrics or Gerontology categories only when the clinical problem addressed by the trials were not exactly recognizable.

\section{5-year Impact factor}

The 2013 5-year journal Impact Factor was extracted by Journal of Citation Report (JCR) (http://thomsonreuters.com/journal-citation-reports/). The 5-year Impact Factor is the average number of times articles from the journal published in the past five years have been cited in the JCR year. Therefore, 2013 5-year Impact Factor covers the period from 2008 to 2012.

\section{Country}

When multicentric studies were performed in different countries, the country of the first author were recorded.

\section{Data analysis}

Comparisons of proportions were analysed by the chisquare test, while the differences in the number of citations for each variable were compared with the one-way ANOVA, with Bonferroni post-hoc in case of variables with more than 2 categories.

Then, data were put in a stepwise multiple regression analysis with citation rate as dependent variable and 
language of publication, indexing in Pubmed database, type of access to articles, subdiscipline, PEDro score, number of authors, country, type of institution, number of centres and 2013 5-year Impact Factor of the publishing journals as independent variables.

In addition, the correlation between PEDro score and the 5-year Impact Factor was analysed with the Spearman's correlation coefficient.

The level of statistical significance was set at 0.05. Data analyses were performed using the SPSS statistical package 12.0 for Windows.

\section{Results}

A total of 674 articles were abstracted in PEDro database. Forty of them were not included in Scopus database and, within the remaining 634, other 15 were not scored in PEDro database. Then, 619 articles, published in 283 journals, were included and analysed. Trials were conducted in 37 countries with no difference in citation rate among them $(p=.919)$. Articles were more frequently written in English language $(93.7 \%, p<.001)$, published with restricted access $(58.5 \%, p<.001)$, listed in PubMed $(93.1 \%, p<.001)$ and included in musculoskeletal subdiscipline $(30.2 \%, p<.001)$; 507 journals had 5 -year Impact Factor and mean 5-year Impact Factor and PEDro score were $3.8 \pm 5.5$ and $6.1 \pm 1.5$, respectively.

The citation rate was significantly higher for articles written in English language, listed in PubMed, and published in journals with a 5-year Impact Factor (Table 1). Post-hoc analyses showed that the citation rate was significantly higher for articles published with delayed open access (vs open access: $p<.001$; vs restricted access: $p=.042$ ) while no significant differences were found between subdisciplines.

The multiple regression analysis showed that the 5year Impact Factor was the strongest independent variable associated with the citation rate $(\beta=4.828 ; 95 \%$ confidence interval $=4.382 ; 5.276$ ), explained approximately $50 \%$ of the variance (adjusted $R^{2}=.503$ ). The number of authors also was independently associated with citation rate $(\beta=1.700 ; 95 \%$ confidence interval $=.894$; $2.506)$, and explained approximately further $1 \%$ of variability (cumulative adjusted $\mathrm{R}^{2}=.516$ ). The other variables were excluded from the model (Table 2).

A significant correlation between PEDro score and 5year Impact Factor of the publishing journal was found (Spearman's correlation coefficient $=.197 ; p<.001$ ).

\section{Discussion}

This study was aimed to test what factors are associated with citation rate of RCTs published on the physiotherapy field.

Evaluating scientific quality of research groups and scientific journals is a notoriously difficult problem that
Table 1 Comparisons of citation rate among articles' characteristics $(n=619)$

\begin{tabular}{|c|c|c|}
\hline Variable & frequency & citatior \\
\hline \multicolumn{3}{|l|}{ Language } \\
\hline English & $580(93.7 \%)$ & $28 \pm 41$ \\
\hline Others & $39(6.3 \%)$ & $3 \pm 4$ \\
\hline \multicolumn{3}{|l|}{ Subdiscipline } \\
\hline Musculoskeletal & $187(30.2 \%)$ & $22 \pm$ \\
\hline Neurology & $91(14.7 \%)$ & $26 \pm$ \\
\hline Cardiothoracics & 89 (14.4\%) & $36 \pm$ \\
\hline Pediatrics & $18(2.9 \%)$ & $19 \pm$ \\
\hline Gerontology & $52(8.4 \%)$ & $31 \pm$ \\
\hline Continence/womens' health & $25(4.0 \%)$ & $16 \pm$ \\
\hline Oncology & $29(4.7 \%)$ & $28 \pm$ \\
\hline Sport & $11(1.8 \%)$ & $28 \pm$ \\
\hline Occupational health & $8(1.3 \%)$ & $16 \pm 9$ \\
\hline Endocrinology & $68(11.0 \%)$ & $38 \pm$ \\
\hline Others & $41(6.6 \%)$ & $14 \pm$ \\
\hline
\end{tabular}

Pubmed

$\begin{array}{lll}\text { yes } & 576(93.1 \%) & 28 \pm 41 \\ \text { no } & 43(6.9 \%) & 5 \pm 5\end{array}$

Countries

Australia
Canada
United Kingdom
USA
Sweden
Brazil
Germany
Turkey
The Netherlands
China
Others

$50(8.1 \%) \quad 34 \pm 58$

$26(4.2 \%) \quad 44 \pm 84$

$57(9.2 \%) \quad 24 \pm 35$

$137(22.1 \%) \quad 32 \pm 34$

$26(4.2 \%) \quad 19 \pm 14$

$29(4.7 \%) \quad 16 \pm 14$

$25(4.0 \%) \quad 19 \pm 19$

$26(4.2 \%) \quad 23 \pm 23$

$35(5.7 \%) \quad 33 \pm 37$

$41(6.6 \%) \quad 19 \pm 58$

167 (27.0\%)

Type of access

\section{Open access \\ Delayed open access}

Restricted access

$79(12.6 \%) \quad 34 \pm 56$

$175(28.9 \%) \quad 37 \pm 59$

$365(58.5 \%) \quad 20 \pm 18$

Number of centres

multicentric

single-centre

$532(85.9 \%) \quad 26 \pm 1$

$87(14.1 \%) \quad 83 \pm 9$

5-year Impact Factor

Yes

No

$507(81.9 \%) \quad 29 \pm 43$

$112(18.1 \%) \quad 12 \pm 16$

Academic setting

$455(73,5 \%) \quad 26 \pm 36$

No

$164(26.5 \%) \quad 26 \pm 49$ 
Table 2 Regression analysis (dependent variable: citation rate)

\begin{tabular}{llll}
\hline & Beta & $t$-test & $P$ value \\
\hline Model: & & & \\
Constant & & -.679 & $<.001$ \\
5 year Impact Factor & .658 & 21.276 & $<.001$ \\
Number of authors & .128 & 4.144 & $<.001$ \\
Excluded variables: & & & \\
PEDro score & .030 & 1.034 & .302 \\
Country & .011 & .390 & .697 \\
Academic & -.005 & -.162 & .871 \\
Multicentric & .041 & 1.321 & .187 \\
Language & .029 & .999 & .318 \\
Subdiscipline & -.017 & -.608 & .544 \\
Pubmed & .034 & 1.191 & .234 \\
Type of access & -.027 & -.907 & .365 \\
\hline
\end{tabular}

has no accepted standard solution. The use of citation frequency as a measure of quality of research is strongly criticized and debated [25, 26]. Anyway, the growing competition for research funding and academic positions, combined with an increasing use of bibliometric parameters to evaluate careers (e.g. number of publications and the impact factor of the journals they appeared in), pressures scientists into continuously producing publishable and citable results [27]. Also Journals and Editors are interested in bibliometric parameters because they may influence subscriptions and where authors submit papers $[28,29]$.

In this work, we considered as independent variables many of the most relevant factors that may reduce the value of citation count as indicator of scientific impact [1].

We found that the 5-year Impact Factor of the original publishing journal was the strongest predictor of citations. This result was not fully expected, since 5-year Impact Factor is a bibliometric indicator for journals, while the citation rate is for individual papers. In fact, Shadgan et al. [30] found that the most frequently cited articles published in the rehabilitation field were not always in the journals with the highest Impact Factor. Also Lozano et al. [31] found that, since 1990, the proportion of highly cited papers coming from highly cited journals has been decreasing, and accordingly, the proportion of highly cited papers not coming from highly cited journals has also been increasing. In addition, the 5-year Impact Factor was extracted from JCR, while the number of citations per article was extracted from Scopus and different citation databases may produce different citation count [32]. Then, these two variables cannot be judged co-dependent, since they are placed on different levels and based on two different databases: an article may be published in a journal with high bibliometric indicators and obtain few citations and, at the same time, a paper published in a journal not indexed in JCR may receive high number of citations within Scopus database. Since PEDro has been shown to be the largest database on physiotherapy field [33, 34], we chose to use Scopus database for the citation count because it includes a larger number of journals (and articles) in comparison to JCR [35], the two most widely used tools for generating citation count. A different option could have been Google Scholar, but its use is largely criticized because it covers and uses for citation count, in addition to print and electronic journals, conference proceedings, books, theses, dissertations and abstracts (i.e. not peerreviewed literature) [32, 35]. Furthermore, when searching for documents citing a specific paper in Google Scholar, a few duplicates can be found because the same article can be extracted from different archives [36].

Callaham et al. [8] found similar results in a study on articles published in the field of Emergency Medicine and indexed in PubMed. In a cohort of 204 published articles, the strongest predictor of citations per year was the Impact Factor of the original publishing journal. Our results suggest that citation may be more strongly influenced by the reputation of the publishing journal than by the quality of research or its accessibility (i.e. electronic searching, language of publication, open access system). This is a well known phenomenon, similar to what Cozzens [37] calls "success-breeds-success" or also known as "Matthew Effect" [38], which is possible not only for highly-cited scientists, but also for highly-cited publications [39].

A different explanation might be that journals with high 5-year Impact Factor publishes high quality studies in any case. This seems to be questionable, since we found a weak correlation between quality (total PEDro score) of RCTs and 5-year Impact Factor of the publishing journal. Costa et al. [40] found no correlation between RCTs quality on physiotherapy interventions, assessed by the PEDro score, and the 2-year Impact Factor of journals where the studies were published. On the basis of this investigation, Authors concluded that the journals 2-year Impact Factor is not a valid measure of RCT quality. Thus, the journal where a paper is published seems to give it more chances to be cited over and above their intrinsic quality or relevance [39].

In previous studies the citation count was performed after 3 [10], 3,5 [8] or 4 years [41] from publication. A period of 5 years may be better suited, especially for clinical journals, for which there is usually a relatively high citation rate up to at least 6 years after publication or even longer [42]. For this reason, the 2013 5-year Impact Factor was chosen as bibliometric indicator. We have to take into account that journals included in JCR can change over time: for example, within the category 
"Rehabilitation", 28 journals were included in the year 2008 (year of publication of RCTs) and 62 in the year 2013 (year of final count of citations).

More surprisingly, methodological quality of RCTs, assessed by PEDro score, was not a predictor of citation rate. Items of PEDro scale assess the internal validity and statistical reporting of articles, while factors related to external validity (e.g. study participant recruitment and selection procedures, choice of outcomes measures) [43] are not included in the scale. The choice of using this type of scale might have played down the weight of the quality of RCTs as predictor in the model. Quality is a complex concept not easy to measure. For example, it has been shown that the citation counts of papers were highly related to clinical utility $[44,45]$.

In our sample, open access system was not a predictor of citation rate. This result is not really surprising, since the real role of the free access to journal articles on their citation rate is still debated. For example, in most studies, it has been suggested that free access articles are cited significantly more times than articles that are not open access [46, 47]. On the other hand, others [48, 49] showed that open access articles received significantly more downloads and audience, but they were not cited more frequently than non-open access articles. In the same way, PubMed indexing, subdisciplines, English language were not predictors of citation rate and this is explainable by the high number of articles having these characteristics (about $95 \%$ ). PubMed database was chosen as independent variable because it is free and provide open access to the records to all interested clinicians, researchers, and trainees and also to the public in general. The association of PEDro score with language of publication and subdisciplines were recently investigated. Despite language of publication (English versus other languages) has been found to be associated with the methodological quality of reports of physiotherapy trials, the magnitude of this influence is small [50]. It has also recently found that methodological quality of trial reports varies according to the subdiscipline of physiotherapy, classified according to the PEDro database classification [17]. The country and the type of institution where the study was performed and the number of centres involved in the study also were not associated to citation rate. Otherwise, Pasterkamp et al. [51] observed that citation frequency may be significantly augmented by nation oriented citation bias. Many of the these variables (open access system, language of publication, subdisciplines, country) are commonly considered sources of bias in using citation rates as a measure of scientific impact [1] and their exclusion from the regression model suggests that their influence is low in our sample.

As found by Beaver [52], the number of authors was associated to citation frequency and it is also considered a sources of bias. However, in our analysis it was a very poor predictor of citation rate.

The study had some limitations, that should be taken into account in the interpretation of results. About $8 \%$ $(n=55)$ of articles were excluded. The use of Scopus may have introduced bias. However, in our sample, Scopus failed to list less than $6 \%$ of trials found in PEDro, and such coverage can be considered acceptable, since PEDro have virtually complete coverage of RCTs in physiotherapy field [33, 34]. In addition, Fell et al. [53] reported that Scopus provide a strong coverage of physical therapy literature. Fifteen articles (about $2 \%$ ) were not scored in PEDro database and excluded from the study because the full text are written in languages that we were not able to translate. Articles published in just one year were included. We know that IF can vary from year to year depending on the published material in a particular year; in fact, a large random component in IF changes has been reported [54].

The inclusion of self-citations is a potential source of bias. However, the exclusion of self-citations is a controversial issue. In fact, one should discriminate between different kinds of author self-citations, from those that are informative to those that are self-enhancing [55].

Finally, a number of variables, such as clinical relevance or newsworthiness, as well as generalisability of results, were not included in the model because they are hard to measure.

\section{Conclusions}

In conclusion, the study highlights that neither accessibility (language of publication, indexing in PubMed database or the type of access to articles) nor reported quality (PEDro score), but 5-year Impact Factor, is a strong predictor of the number of citations for RCTs in the physiotherapy field. Our findings support the increasingly widespread idea that citation analysis does not reflect the scientific merit of the cited work, at least in terms of reported quality. In our sample, the main threat is the so called "Matthew Effect".

The results of this study need to be confirmed by studies on a publication time longer than one year.

\section{Abbreviations \\ RCT: Randomised controlled trial; JCR: Journal citation report; PEDro: Physiotherapy evidence database.}

\section{Competing interests}

The authors declare that they have no competing interests.

\section{Authors' contributions}

MP conceptualized and designed the study, analysed and interpreted the data, drafted the manuscript, and approved the final manuscript as submitted. $\mathrm{NL}$ acquired data, drafted the initial manuscript and approved the final manuscript as submitted. GB acquired data, critically reviewed and revised the manuscript, and approved the final manuscript as submitted. BL conceptualized the study, critically reviewed and revised the manuscript for important intellectual content and approved the final manuscript as submitted. 


\section{Author details}

${ }^{1}$ Unit of Functional Rehabilitation, Department of Continuing Care, Azienda USL 4, Prato, Italy. ${ }^{2}$ Private practice, Firenze, Italy. ${ }^{3}$ RCCS Fondazione Don Gnocchi, Firenze, Italy.

Received: 22 April 2015 Accepted: 5 August 2015 Published online: 01 September 2015

\section{References}

1. Bornmann L, Daniel H-D. What do citation counts measure? A review of studies on citing behavior. J Docum. 2008;64:45-80.

2. Thonon F, Boulkedid R, Delory T, Rousseau S, Saghatchian M, van Harten W, et al. Measuring the outcome of biomedical research: a systematic literature review. PLoS One. 2015;10:e0122239.

3. Radicchi F, Fortunato S, Castellano C. Universality of citation distributions: Toward an objective measure of scientific impact. Proc Natl Acad Sci U S A. 2008;105:17268-72.

4. Gatti R, Paci M, Vercelli S, Baccini M. Has the Italian Academia missed an opportunity? Phys Ther. 2014;94:1358-60.

5. Ahlgren P, Colliander C, Persson O. Field normalized citation rates, field normalized journal impact and Norwegian weights for allocation of university research funds. Scientometrics. 2012;92:767-80.

6. Kratz JE, Strasser C. Researcher perspectives on publication and peer review of data. PLoS One. 2015;10:e0117619.

7. Buela-Casal G, Zych I. What do the scientists think about the impact factor? Scientometrics. 2012;92:281-92.

8. Callaham M, Wears RL, Weber E. Journal prestige, publication bias, and other characteristics associated with citation of published studies in peer-reviewed journals. JAMA. 2002;287:2847-50.

9. Kulkarni AV, Busse JW, Shams I. Characteristics associated with citation rate of the medical literature. PLoS One. 2007;2:e403.

10. Bhandari M, Busse J, Devereaux PJ, Montori VM, Swiontkowski M, Tornetta lii $P$, et al. Factors associated with citation rates in the orthopedic literature. Can J Surg. 2007:50:119-23.

11. Patsopoulos NA, Analatos AA, loannidis JP. Relative citation impact of various study designs in the health sciences. JAMA. 2005;293:2362-6.

12. Figg WD, Dunn L, Liewehr DJ, Steinberg SM, Thurman PW, Barrett JC, et al. Scientific collaboration results in higher citation rates of published articles. Pharmacotherapy. 2006;26:759-67.

13. Kocak FU, Unver B, Karatosun V. Level of evidence in four selected rehabilitation journals. Arch Phys Med Rehabil. 2011;92:299-303.

14. Paci M, Cigna C, Baccini M, Rinaldi LA. Types of article published in physiotherapy journals: a quantitative analysis. Physiother Res Int. 2009;14:203-12.

15. Paci M, Briganti G, Lombardi B. Levels of evidence of articles published in Physical and Rehabilitation Medicine journals. J Rehabil Med. 2011;43:264-7.

16. Moseley AM, Herbert RD, Maher CG, Sherrington C, Elkins MR. Reported quality of randomized controlled trials of physiotherapy interventions has improved over time. J Clin Epidemiol. 2011;64:594-601.

17. Moseley AM, Elkins MR, Janer-Duncan L, Hush JM. The quality of reports of randomized controlled trials varies between Subdisciplines of Physiotherapy. Physiother Can. 2014;66:36-43.

18. Paci M, Landi N, Marchettini M, Baccini M. Randomized controlled trial quality in pediatric physical therapy. Phys Occup Ther Pediatr. 2014;34:260-70.

19. Moseley AM, Herbert RD, Sherrington C, Maher CG. Evidence for physiotherapy practice: a survey of the physiotherapy evidence database (PEDro). Aust J Physiother. 2002;48:43-9.

20. Sherrington C, Herbert RD, Maher CG, Moseley AM. PEDro: a database of randomized trials and systematic reviews in physiotherapy. Man Ther. 2000;5:223-6.

21. Maher CG, Sherrington C, Herbert RD, Moseley AM, Elkins M. Reliability of the PEDro scale for rating quality of randomized controlled trials. Phys Ther. 2003;83:713-21.

22. de Morton NA. The PEDro scale is a valid measure of the methodological quality of clinical trials: a demographic study. Aust J Physiother. 2009;55:129-33.

23. Macedo LG, Elkins MR, Maher CG, Moseley AM, Herbert RD, Sherrington C. There was evidence of convergent and construct validity of physiotherapy evidence database quality scale for physiotherapy trials. J Clin Epidemiol. 2010;63:920-5
24. Olivo SA, Macedo LG, Gadotti IC, Fuentes J, Stanton T, Magee DJ. Scales to assess the quality of 320 randomized controlled trials: A systematic review. Phys Ther. 2008;88:156-75.

25. Seglen PO. Why the impact factor of journals should not be used for evaluating research. BMJ. 1997;314:498-502.

26. Pendlebury DA. The use and misuse of journal metrics and other citation indicators. Arch Immunol Ther Exp (Warsz). 2009;57:1-11.

27. Fanelli D. Do pressures to publish increase Scientists' bias? An Empirical Support from US States Data PLoS ONE . 2010;5:e10271.

28. Smith R. Commentary: the power of the unrelenting impact factor-is it a force for good or harm? Int J Epidemiol. 2006;35:1129-30.

29. Ogden TL, Bartley DL. The Ups and Downs of Journal Impact Factors. Ann Occup Hyg. 2008;52:73-82.

30. Shadgan B, Roig M, HajGhanbari B, Reid WD. Top-cited articles in rehabilitation. Arch Phys Med Rehabil. 2010;91:806-15.

31. Lozano GA, Larivière $V$, Gingras $Y$. The weakening relationship between the Impact Factor and papers' citations in the digital age. J Am Soc Inf Sci. 2012;63:2140-5.

32. Kulkarni AV, Aziz B, Shams I, Busse JW. Comparisons of citations in Web of science, Scopus, and Google scholar for articles published in general medical journals. JAMA. 2009;302:1092-6.

33. Moseley AM, Sherrington C, Elkins MR, Herbert RD, Maher CG. Indexing of randomised controlled trials of physiotherapy interventions: a comparison of AMED, CENTRAL, CINAHL, EMBASE, Hooked on Evidence, PEDro. PsycINFO PubMed Physiotherapy. 2009;95:151-6.

34. Michaleff ZA, Costa LO, Moseley AM, Maher CG, Elkins MR, Herbert RD, et al. CENTRAL, PEDro, PubMed, and EMBASE are the most comprehensive databases indexing randomized controlled trials of physical therapy interventions. Phys Ther. 2011;91:190-7.

35. Falagas ME, Pitsouni El, Malietzis GA, Pappas G. Comparison of PubMed, Scopus, Web of science, and Google scholar: strengths and weaknesses. FASEB J. 2008;22:338-42.

36. Jacso P. As we may search: comparison of major features of the Web of science, Scopus, and Google scholar citation-based and citation-enhanced databases. Curr Sci. 2005;89:1537-47.

37. Cozzens SE. Comparing the sciences - citation context analysis of papers from neuropharmacology and the sociology of science. Soc Stud Sci. 1985;15:127-53.

38. Merton RK. The Matthew effect in science. Science. 1968;159(3810):56-63.

39. Larivière $V$, Gingras $Y$. The impact factor's Matthew effect: a natural experiment in bibliometrics. J Am Soc Inf Sci. 2010;61:424-7.

40. Costa LO, Moseley AM, Sherrington C, Maher CG, Herbert RD, Elkins MR. Core journals that publish clinical trials of physical therapy interventions. Phys Ther. 2010;90:1631-40.

41. Willis DL, Bahler CD, Neuberger MM, Dahm P. Predictors of citations in the urological literature. BJU Int. 2011;107:1876-80.

42. Grimby G. Invited commentary on "Level of evidence in four selected rehabilitation journals.". Arch Phys Med Rehabil. 2011;92:304-5.

43. Steckler A, McLeroy KR. The importance of external validity. Am J Public Health. 2008;98:9-10

44. Fenton JE, O'Connor A, Ullah I, Ahmed I, Shikh M. Do citation classics in rhinology reflect utility rather than quality. Rhinology. 2005;43:221-4.

45. Lokker C, McKibbon KA, McKinlay RJ, Wilczynski NL, Haynes RB. Prediction of citation counts for clinical articles at two years using data available within three weeks of publication: retrospective cohort study. BMJ. 2008;336:655-7.

46. Eysenbach G. Citation advantage of open access articles. PLoS Biol. 2006;4:e157.

47. Gargouri Y, Hajjem C, Lariviére V, Gingras Y, Carr L, Brody T, et al. Selfselected or mandated, open access increases citation impact for higher quality research. PLoS One. 2010;5:e13636.

48. Davis PM. Open access, readership, citations: a randomized controlled trial of scientific journal publishing. FASEB J. 2011;25:2129-34.

49. Björk BC, Solomon D. Open access versus subscription journals: a comparison of scientific impact. BMC Med. 2012;10:73.

50. Shiwa SR, Moseley AM, Maher CG, Pena Costa LO. Language of publication has a small influence on the quality of reports of controlled trials of physiotherapy interventions. J Clin Epidemiol. 2013;66:78-84.

51. Pasterkamp G, Rotmans JI, de Kleijn DVP, Borst C. Citation frequency: A biased measure of research impact significantly influenced by the geographical origin of research articles. Scientometrics. 2007;70:153-65. 
52. Beaver DB. Does collaborative research have greater epistemic authority? Scientometrics. 2004;60:399-408.

53. Fell DW, Burnham JF, Buchanan MJ, Horchen HA, Scherr JA. Mapping the core journals of the physical therapy literature. J Med Libr Assoc. 2011;99:202-7.

54. Garfield E. How can impact factors be improved? BMJ. 1996;313:411-3.

55. Hartley J. To cite or not to cite: Author self-citations and the impact factor. Scientometrics. 2012;92:313-7.

Submit your next manuscript to BioMed Central and take full advantage of:

- Convenient online submission

- Thorough peer review

- No space constraints or color figure charges

- Immediate publication on acceptance

- Inclusion in PubMed, CAS, Scopus and Google Scholar

- Research which is freely available for redistribution 